\title{
A Study on the Stability Improvement Technique of Electric Walkers to Prevent Falls of the Elderly
}

\author{
Munsuck Jang ${ }^{1}$, Woosu Kim ${ }^{2}$, Seungjin Yoon ${ }^{2}$ and Eunghyuk Lee ${ }^{1}$ \\ ${ }^{1}$ Dept. Of Electronic Engineering, Korea PolyTechnic Univ., Gyeonggi-Do, Korea \\ ${ }^{2}$ Korea Evaluation Institute of Industrial Technology \\ \{msjang,ehlee\}@kpu.ac.kr, \{kws,sjyoon\}@keit.re.kr
}

\begin{abstract}
Although the elderly with lower extremity muscle strength has been supported by electric walkers for their smooth walking activities, the electric walkers do not guarantee the stability of outdoor activities due to lack of walking stability and convenience. Also, inexperiences in operating an electric walker during actual walking may cause falls because of existing a separation distance between a user and an electric walker. In this study the center of gravity of a user and the safety area are determined using the separation distance between a user and an electric walker and an algorithm that minimizes falls through a walking synchronization between a user and an electric walker is proposed. In experiment results, the safety area is configured by $40 \sim 80 \mathrm{~cm}$ based on its normal distribution for subjects with different physical conditions. It is possible to reduce the standard error, 37\%, according to use of a synchronization controller in walking movements and to ensure the stability of subjects through reducing the speed of $0.45 \mathrm{~m} / \mathrm{s}$ maximum.
\end{abstract}

Keywords: Walking assist, Fall, Laser Range Finder, Stability

\section{Introduction}

Movements in retirement living, such as hearing, sight, conversation, walking, meal, washing, and etc., have to be ensured as essential factors for happy life of the elderly. After the 60s average, however, reflexes and muscle strengths are decreased and bone densities are also significantly decreased. It brings easy falls caused by a light shock and that causes fractures [1].

Falls of the elderly become one of dangerous accidents that affects the quality of retirement living. The elderlies age over 65 went through falls about $10 \sim 30 \%$. About $30 \%$ of them experienced damages, which require medical attentions, like scratches and sprains and about $10 \%$ of them went through fractures and that leads to inhibit physical functions, movements, and independencies of the elderly [2].

For managing easy and convenient living in the elderly, it is necessary to ensure their walking without any inconveniences. Although most of the elderly who represent lower extremity muscle strength have been supported by manual walking assists (walkers), such as a four-point stick or pram [3], uses of electric walkers have been increased. However, recently used electric walkers cause accidents like falls due to lack of stability and convenience in the walking of the elderly.

Recently studies on detecting and preventing falls of the elderly have been conducted. Kim [4] proposed a method that predicts falls through collecting postures of the elderly using acceleration sensors and storing its data to DB. However, it was only conducted as a function that detects falls of the elderly and notifies it to their guardians. Although Hwang [5] proposed a method that detects falls with real-time using acceleration and geo-magnetic sensors installed in a smart phone, it is different from the issue of preventing falls because it is a resolving 
action after the accident. The RT-Walker [6] proposed by Yasuhisa estimated the center of gravity $(\mathrm{CoG})$ using a human modeling method through detecting seven joints using a laser range finder (LRF) for the sagittal plane in a user. The efficiency of the RT-Walker was verified by a motion capture system and it proposed a method that prevents movements and falls through estimating $\mathrm{CoG}$ with real-time. However, it showed a disadvantage that requires the preliminary information of user joints before estimating CoG. Yoshihiro Kai [7] introduced a method that detects the positions of the feet of Parkinson's patients using IR sensors installed at a walker. However, it is not suitable for outdoor environments because it is a type of walking table even though it can prevent front falls through a sudden stop of the walker as the positions of feet are not detected.

In this study the causes of falls in the elderly are analyzed for improving the problems mentioned above and the environment and factor that cause falls during the operation of an electric walker are investigated. Then, a safety region is determined by deriving $\mathrm{CoG}$ using the separation distance between the electric walker and a user. Also, a method that minimizes falls through synchronizing the speed of the walker and the walking speed of a user based on the safety region is proposed.

\section{Causes of Falls in Operating an Electric Walker}

\subsection{Analysis of Falls in the Elderly}

The elderly get hurt from fall and slipping accidents easily due to their physical conditions, such as decreases in balances, degenerations in nerve systems, decreases in walking capabilities, weakening muscle strengths, and etc., according to aging. Based on a statistic, falls in the elderly occur frequently in indoor environments like stairs, bathrooms, bedrooms, and so on. The major causes in the falls are divided into external causes, such as false steps, slipping, leg folds, and etc., and internal causes, such as side effects of drugs, dizziness, and so on [8]. In the results of analyzing the place and cause of falls, it is necessary to ensure convenient walking, sitting, and standing and to prevent such external causes of falls in order to manage everyday life. It is also presented in the item that measures the balance, standing from a chair, and walking speed in a campaign program for preventing falls in order to improve the health and function in the elderly [9].

For preparing an aging society and improving the quality of life in the elderly, studies on preventing falls of the elderly have been conducted by ensuring a wide supporting plane using a rehabilitation assist device as a core device that combines a manual walking assist device and IT[10] and RT technologies for the elderly with low muscle strengths.

\subsection{Problems in Electric Walkers and its Solutions}

A general walking aid has two or more wheels and represents a structure that supports the front or side of the human body and the weight during the movement of the walking aid. The purpose of the walking aid is to ensure the stability of users who have difficulties in standing or walking due to lowering muscle strengths and to extend their moving distances. In the case of the manual walking aid, however, it may cause falls at slops (rough roads) due to lowering muscle strengths and there are risks of falls because of overturning the walking aid in actions of standing and sitting [11]. For improving these problems, a study on an electric walker that can walk through a minimum handling in slops by installing an electric motor to such a manual device has performed [12]. However, the electric walker shows a limitation of operating the device accurately according to the intension of a user compared to 
that of the manual device [13]. In particular, in the case of using the electric walker by the elderly it may cause falls due to the separation distance between the device and the user because of lowering both the motor nerves and the cognition capabilities.

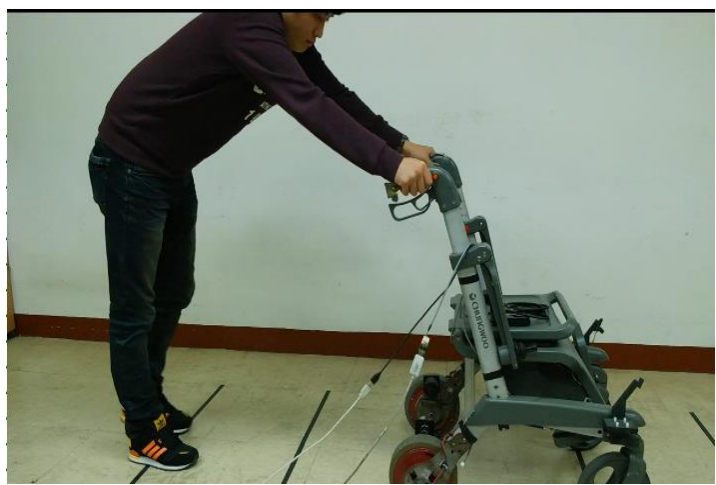

Figure 1. Cause of Falls

In the results of walking experiments using an actual electric walker shown in Figure 1, there may occur a front fall because of collapsing CoG as the user misses the handle due to the fact that there exists a separation distance between the electric walker and the user if the user is supported by the horizontal handle bar. Thus, in this study the distance between the electric walker and the user is analyzed to solve this problem and a safety region is determined. Then, a method that prevents externally caused falls by maintaining the balance through a synchronization between the speed of the electric walker and the user walking speed is proposed.

\section{Speed Control Method for Preventing Falls}

\subsection{CoG for the Walking Stability}

In general, for improving the stability of an object different methods such as an increase in weight, an increase in the friction force between an object and a contact surface, an increases in the base of support along the direction of the external force, an arrangement of the horizontal position based on the center of gravity to the edge of the base of support, and a lowering of the center of gravity are used [14]. The most important factor for determining the walking stability is the center of gravity (CoG) [15].

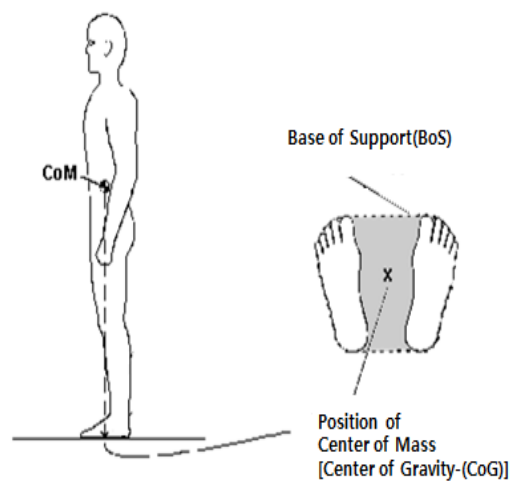

Figure 2. Factor of Determining the Stability 
As shown in Figure. 2, CoG is located above the feet vertically and is collapsed as it is contrary to the vertical line. Thus, the wider the support area, the higher the safety. As shown in Figure 3, the CoG of the user is measured by installing a laser scanner to the electric walker in which (a) and (b) show the knee joint positions of the user and (c) represents the CoG. The effective range of the laser scanner was determined by $60^{\circ}$ and the output data of the laser scanner can be presented as a form of $r_{\text {sensor }}(i)$ for the rotational angle, $\theta_{\text {scan }}$, as noted in equation (1).

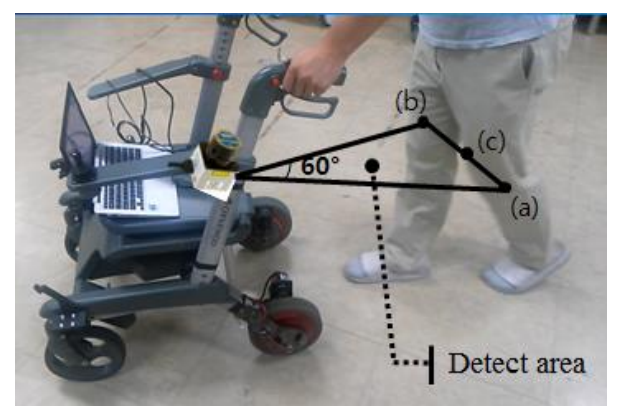

Figure 3. Effective Range of the Laser Scanner

$$
\begin{array}{ll}
\mathrm{r}_{\text {sensor }}(\mathrm{i}), & \mathrm{i}=1,2,3 \ldots, 170(\mathrm{~m} \mathrm{~m}) \\
\theta_{\text {scan }}(i)=i \times \Delta \theta_{\text {scan }}, & \Delta \theta_{\text {scan }}=0.36^{\circ}
\end{array}
$$

Using the laser scanner the diagonal distance between the electric walker and the user can be measured. The coordinate for each measurement point can be presented using $\theta_{\text {scan }}$ as equation (2) [16].

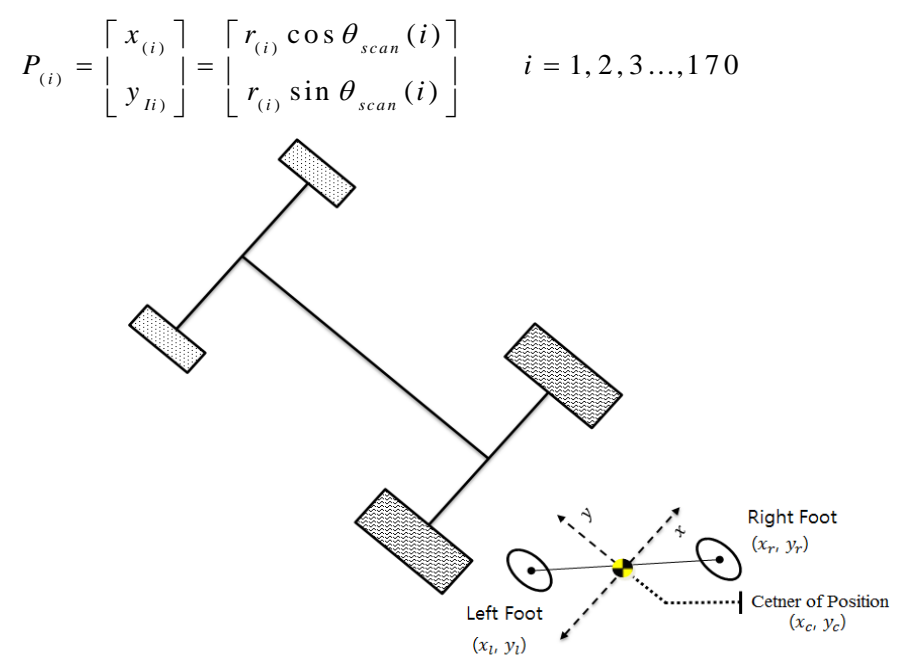

Figure 4. Detection of the Center of the User

It can be presented as a tangent point between the laser scanner and a knee joint and be expressed as a two dimensional coordinate. Also, it is possible to calculate $\left(x_{l}, y_{l}\right)$ and $\left(x_{r}, y_{r}\right)$ using the intermediate value of the two dimensional coordinate of the left and right knee joints as shown in Figure 4. Then, the center point of the user can be obtained using equation (3).

$$
\mathrm{x}_{c}=\frac{x_{l}+x_{r}}{2}, \mathrm{y}_{c}=\frac{y_{l}+y_{r}}{2}(\mathrm{~mm})
$$


Figure 5 represents the coordinate of the center of the user in which the $\mathrm{x}$ and $\mathrm{y}$ axes show the position $(\mathrm{mm})$ of the laser scanner and the distance to the user $(\mathrm{mm})$ respectively. That is, the area, (a), shows the position of (a) is located at the laser scanner $(-73 \mathrm{~mm}, 564 \mathrm{~mm}$ ) and the area, (b), shows the position of (b) is located at the laser scanner $(81 \mathrm{~mm}, 772 \mathrm{~mm})$. Regarding the area $(\mathrm{c})$, the center of the user can be calculated as $(3,668)$ using equation $(3)$.

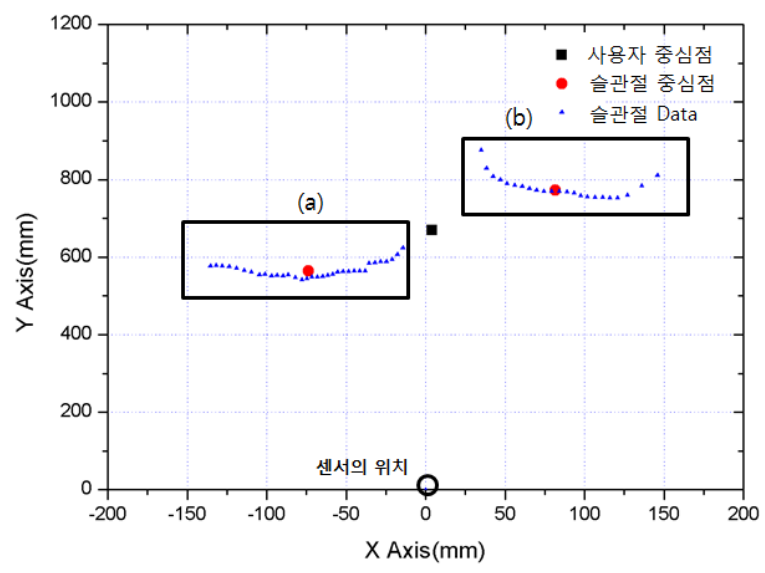

Figure 5. Coordinate of the Center of the User

However, the safety distance between the electric walker and the user is not defined. Thus, the average and standard deviation between the electric walker and the user are to be calculated and its normal distribution can be obtained using equations (4) and (5). Then, the safety region, $\left(S_{x}, S_{y}\right)$, can be calculated based on the distance that represents a low probability.

$$
\begin{aligned}
& S_{y}=\frac{1}{\sigma \sqrt{2 \pi}} \exp \left[-\frac{1}{2}\left(\frac{(y-\mu}{\sigma}\right)^{2}\right] \quad(-\infty<y<\infty ; \sigma>0) \\
& S_{x}=\frac{1}{\sigma \sqrt{2 \pi}} \exp \left[-\frac{1}{2}\left(\frac{(x-\mu}{\sigma}\right)^{2}\right] \quad(-\infty<y<\infty ; \sigma>0)
\end{aligned}
$$

\subsection{Synchronization between the User and the Electric Walker}

The safety region was configured by deriving the center of the user based on the distance between the user and the electric walker. For controlling the speed using LRF, the user walking speed is to be obtained using both the coordinate of the center of the user and the movement time. Figure 6 shows the controller for obtaining the user walking speed. 


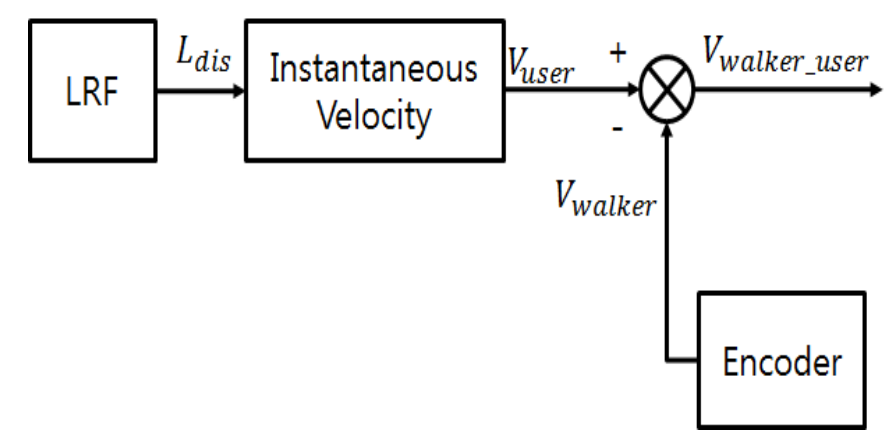

$L_{d i s}:$ Center distance between the electric walker and the user[mm]

$V_{u s e r}:$ Walking speed of the user $[\mathrm{mm} / \mathrm{s}]$

$V_{\text {walker }}:$ Speed of the electric walker $[\mathrm{m} / \mathrm{h}]$

$V_{\text {walker_user }}:$ Synchronization speed $[\mathrm{m} / \mathrm{s}]$

Figure 6. Speed Synchronization Controller

The walking speed of the user $\left(V_{\text {user }}\right)$ can be obtained using the differentiation of the center distance of the user, $L_{d i s}$, and the movement time as shown in equation (6).

$V_{u s e r}=\frac{L_{d i s}}{d t}(m / s)$

To perform the traveling needed by the user, the motion analysis of the device is required according to the input speed of the device. Thus, a kinematic analysis that defines the relationship between control variables and the position and speed using an encoder is precedently performed [17].

A correction variable, $S_{\text {velocity }}$, presented in equation (7) is added to control the speed within the safety region using the speed difference between the user and the electric walker and the speed of electric walker can be varied for the walking speed of the user according to variations in the correction variable.

$V_{\text {wal ker_user }}=\left(V_{\text {user }}-V_{\text {waker }}\right) \times S_{\text {Velocity }}(\mathrm{m} / \mathrm{s})$ 


\section{System Implementation and Experiment}

\subsection{Specification of the Electric Walker}

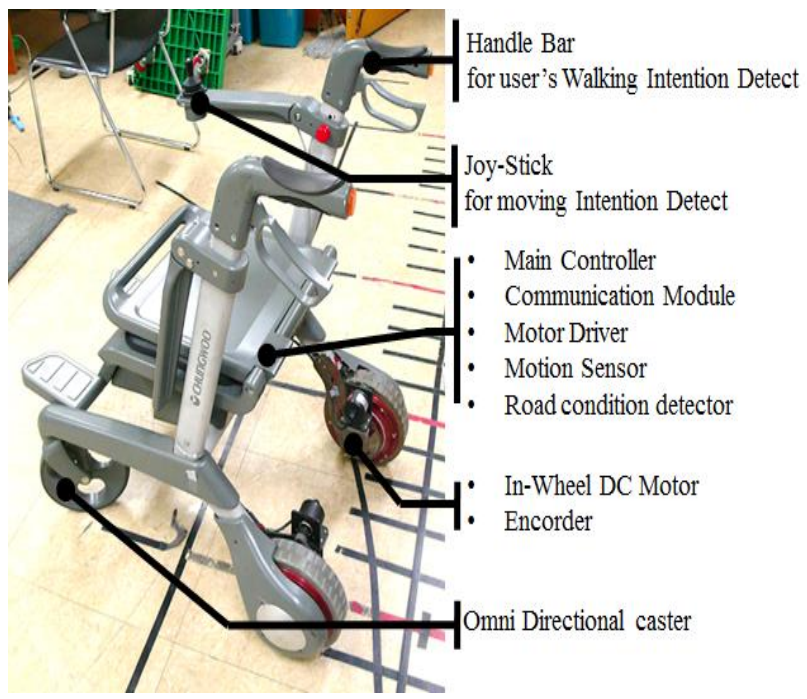

Figure 7. Configuration of the Electric Walker

Table 1. Specification of the Electric Walker

\begin{tabular}{l|l|l}
\hline \multicolumn{1}{c|}{ Item } & \multicolumn{1}{c}{ Specification } \\
\hline \multicolumn{2}{l|}{ Size(W·H·D) } & $630 \times 950 \times 860 \mathrm{~mm}$ \\
\hline Weight & Type & In-wheel DC Motor \\
\hline \multirow{3}{*}{ Motor } & Power & $180 \mathrm{~W} / 24 \mathrm{~V}$ \\
\cline { 2 - 3 } & Diamete & $210 \mathrm{~mm}$ \\
\hline & $\mathrm{r}$ & \\
\hline
\end{tabular}

The electric walker represents a two-wheel operation type and has a wide support area for providing stable supports and boarding to users differed from regular mobile devices. Also, various input devices for detecting user walking and different sensors for measuring motions are installed as shown in Figure 7. Table 1 shows its specification.

The electric walker used in this experiment consists of different modules, such as a user input device that detects walking intension, a user position detection device that synchronizes the speed between the user and the electric walker, a main controller that controls motions input by the walking intension of a user, an actuator section that controls motors according to the output signals of the controller, a road condition sensor device that detects road conditions, a communication device that connects a robot motion sensor device, which detects movement conditions of the robot, and a PC, and a PC program that checks the controller and performs remote control.

\subsection{Experiment for the Derivation of a Safety Region}

As the safety distance regarding the separation distance between the user and the electric walker was not determined precedently, the experiment on the separation distance between the user and the electric walker was implemented for three 
subjects who have different physical conditions as shown in Table 2. The experiment was performed by 10 times in an indoor condition as represented in Figure 8 and the average and standard deviation were obtained. Then, a safety region is proposed based on a distance that shows a low probability through its normal distribution.

Table 2. Subjects of the Experiment

\begin{tabular}{|c|c|c|}
\hline Subject & Sex & Height $(\mathrm{cm})$ \\
\hline A & Female & $161 \mathrm{~cm}$ \\
\hline B & Male & $172 \mathrm{~cm}$ \\
\hline C & Male & $185 \mathrm{~cm}$ \\
\hline
\end{tabular}
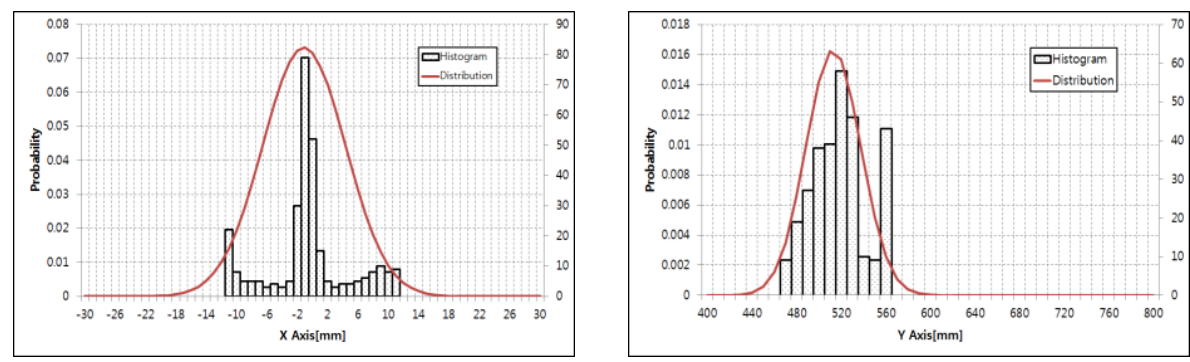

Figure 8. User Center Point Histogram and Normal Distribution of the Subject A

Figure 8 shows the histogram and normal distribution of the subject with a height of $161 \mathrm{~cm}$. The $\mathrm{x}$ axis represents the distribution of the horizontal distance between the laser scanner and the user in which the detected range was $-15 \sim 12[\mathrm{~mm}]$. The $y$ axis shows the vertical distance between the laser scanner and the user in which the detected rang was $460 \sim 560[\mathrm{~mm}]$. Also, the average and standard deviation of $\mathrm{x}$ and $\mathrm{y}$ axes were $-1.1 / 5.4[\mathrm{~mm}]$ and $513 / 24[\mathrm{~mm}]$ respectively. Based on the data, the $\mathrm{x}$ and $y$ axes of the normal distribution represented its safety regions as $-22 \sim 22[\mathrm{~mm}]$ and $400 \sim 620[\mathrm{~mm}]$ respectively.
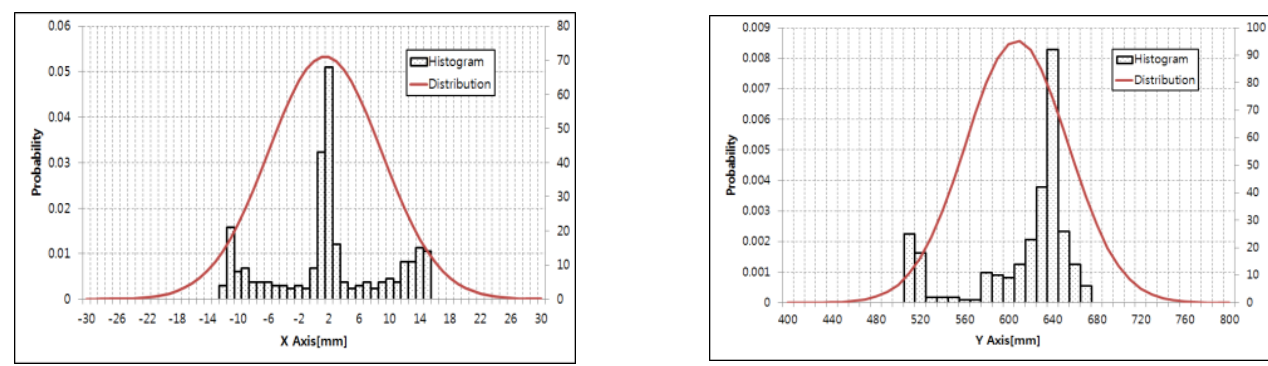

\section{Figure 9. User Center Point Histogram and Normal Distribution of the Subject B}

Figure 9 shows the histogram and normal distribution of the subject with a height of $172 \mathrm{~cm}$. The detections of $\mathrm{x}$ and $\mathrm{y}$ axes were detected as $-13 \sim 15[\mathrm{~mm}]$ and $500 \sim$ $670[\mathrm{~mm}]$ respectively in which the average and standard deviation of $\mathrm{x}$ and $\mathrm{y}$ axes were $1.47 / 7.4[\mathrm{~mm}]$ and $607 / 46[\mathrm{~mm}]$ respectively. It reveals that the $\mathrm{x}$ and $\mathrm{y}$ axes of 
the normal distribution represented its safety regions as $-26 \sim 26[\mathrm{~mm}]$ and $440 \sim 760[\mathrm{~mm}]$ respectively.
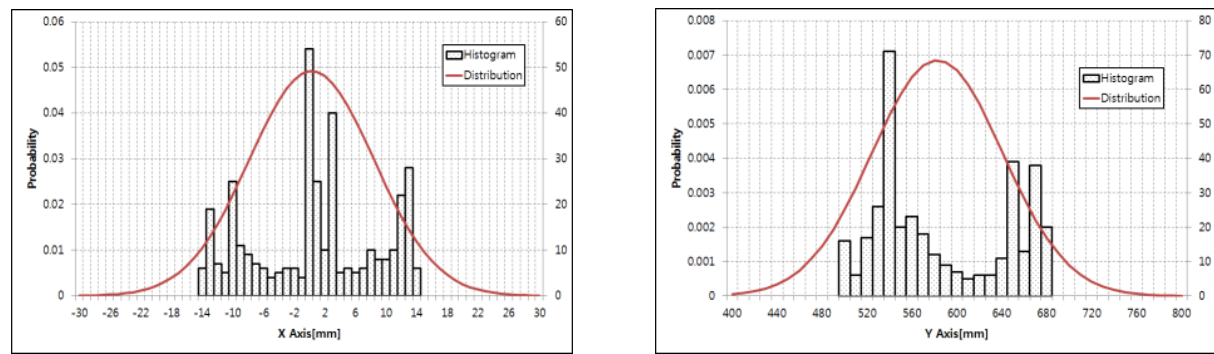

\section{Figure 10. User Center Point Histogram and Normal Distribution of the Subject C}

Figure 10 shows the histogram and normal distribution of the subject with a height of $180 \mathrm{~cm}$. In the case of this subject, the $\mathrm{x}$ and $\mathrm{y}$ axes of the normal distribution represented its safety regions as $-30 \sim 30[\mathrm{~mm}]$ and $400 \sim 800[\mathrm{~mm}]$ respectively.

\subsection{Experiment for Synchronization of the Speed}

For synchronizing the speeds of the user and the electric walker, there are some limitations in producing an actual fall in order to implement an analysis of the measurement of the distance and speed of the user. Thus, as shown in Figure 11 a virtual situation was configured for evaluating the performance of the controller by analyzing the separation distance and speed according to use or unuse of the correction controller.

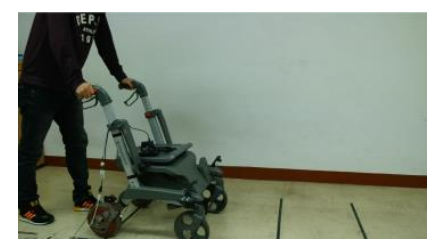

$2[\mathrm{sec}]$

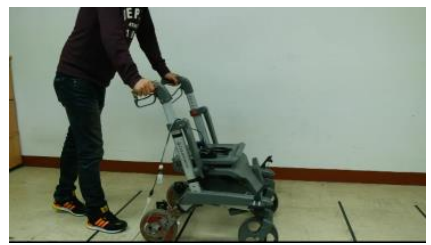

$3[\mathrm{sec}]$

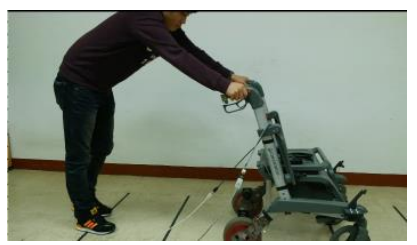

$4[\mathrm{sec}]$

Figure 11. Virtual Situation for Estimating a Fall

Figure 12 shows the center point of the user according to use or unuse of the correction controller mentioned in Figure 6. Figure 13 shows movement speeds where $\mathrm{x}$ and $\mathrm{y}$ axes represent time and speed respectively. In the case of the unuse of the controller, as shown in Figure 12 (a) the distance to the user at the region (1) (4 5 seconds) is detected by $(120,1137)[\mathrm{mm}]$ and that may cause a fall as the speed of electric walker is increased up to $0.76[\mathrm{~m} / \mathrm{s}]$ maximum. In the case of the use of the controller, however, as shown in Figure 12 (b) the distance to the user is decreased by $(20,800)[\mathrm{mm}]$ and the speed of the electric walker is also decreased as $0.13 \sim 0.27[\mathrm{~m} / \mathrm{s}]$. It is verified that the stability of the electric walker is improved through synchronizing the speed between the electric walker and the user. 


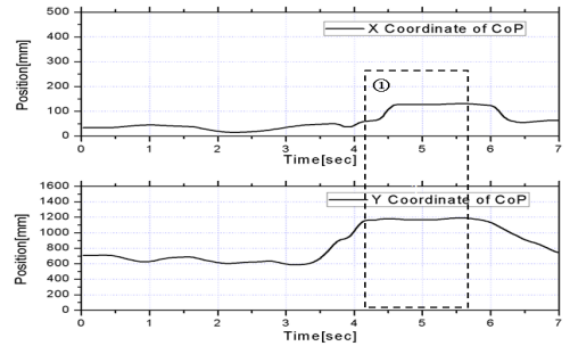

(a) Unuse of the Controller

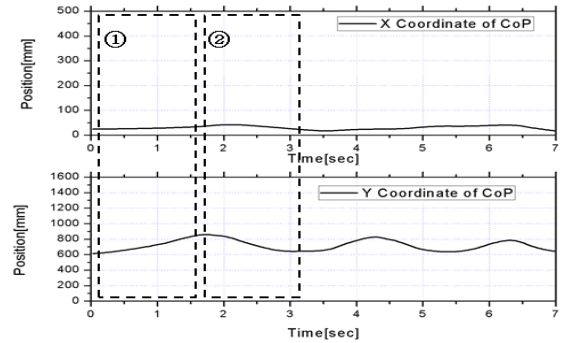

(b) Use of the Controller

\section{Figure 12. Comparison of the User Center Point According to Use or Unuse of the Controller}

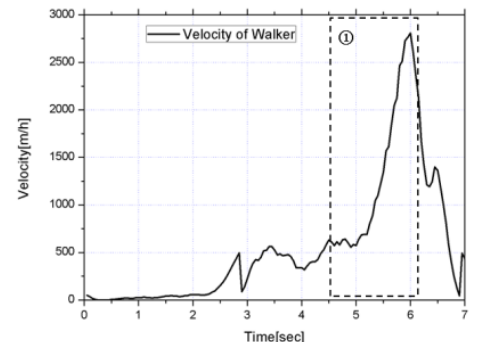

(a) Unuse of the controller

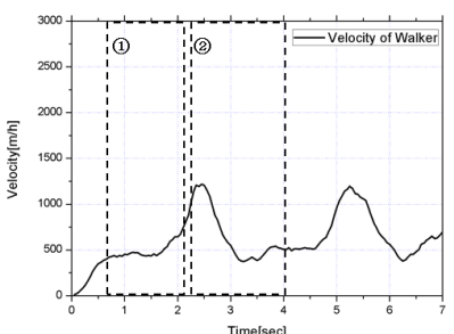

(b) Use of the controller

Figure 13. Comparison of the Speed According to Use or Unuse of the Synchronization Controller

Table 3. Comparision of the Data According to Use or Unused of the Controller

\begin{tabular}{|c|c|c|c|c|c|c|}
\multirow{2}{*}{ State } & \multicolumn{2}{|c|}{ Average (mm) } & \multicolumn{2}{c|}{$\begin{array}{c}\text { Standard Deviation } \\
(\mathrm{mm})\end{array}$} & \multicolumn{2}{c|}{$\begin{array}{c}\text { Standard Error } \\
(\mathrm{mm})\end{array}$} \\
\cline { 2 - 7 } & $\mathrm{X}$ & $\mathrm{Y}$ & $\mathrm{X}$ & $\mathrm{Y}$ & $\mathrm{X}$ & $\mathrm{Y}$ \\
\hline \hline Unuse & 61.62 & 821.32 & 33.67 & 215.73 & 1.84 & 11.54 \\
\hline Use & 3.12 & 716.01 & 12.09 & 68.90 & 0.73 & 4.00 \\
\hline
\end{tabular}

Table 3 shows the comparison of the average, standard deviation, and standard error according to use of the controller. In the case of the unuse of the controller, the error in $\mathrm{x}$ and $\mathrm{y}$ axes were 1.11 and $7.54 \mathrm{~mm}$ and the error rate was $37 \%$.

\section{Conclusion}

In this study a prevention method of falls, which are caused by lowering both the motor nerves and the cognition capabilities of the elderly, in walking motions using an electric walker was proposed. The method configures a safety region through determining the separation distance between the user and the electric walker and measures the speed of the electric walker using encoders. Then, this method synchronizes the speed between the user and the electric walker in order to prevent falls. The experiment was applied to the subjects with different physical conditions and was implemented by measuring the separation distance between the user and the electric walker. Then, a safety region that shows a low probability was determined by measuring the separation distance between the user and the electric walker in which the controller type was varied as the value is below $40 \mathrm{~cm}$ by recognizing it as standing/sitting motions. Based on it, the walking experiment was processed and 
improvements in the stability of the device were verified through synchronizing the speed between the electric walker and the user as the proposed controller was used.

Based on the results of this study, it is necessary to conduct a study on implementing an electric walker using IR sensor arrays, ultra sonic sensor arrays, and etc., which can be used to practical products, instead of using an expensive laser scanner.

\section{Acknowledgement}

This research was supported by the MSIP(Ministry of Science, ICT and Future Planning), Korea, under the C-ITRC(Convergence Information Technology Research Center)support program(IITP-2015-H8601-15-1003) supervised by the IITP(Institute for Information \& communications Technology Promotion).

\section{References}

[1] K. H. Jeon, "The 2004 Survey on the Living Profile and Welfare Service Needs of Older Persons: Results and Ploicy Implications", The Korea Institute for Health and Social Affairs(KIHASA) Health welface Form, (2005), pp.50-56.

[2] M. J. Kim, "Associated factors caused by falls of older people in community-dwelling", Unpublished master's theis, Ewha Woman's University, (2004).

[3] T. Tamura, M. Sekine, H. Kuno, M. Fujie, A. Mori, K. Andoh, "Evaluation of Walkers for Elderly People", Proceedings of the 23rd Annual EMBS International Conference, (2001) October 1391-1392.

[4] N.S. Kim, "An Efficient Methodology of Fall Detection for Ubiquitous Healthcare", Journal of Advanced Information Technology and Convergence, vol. 8, no. 8, (2010).

[5] S.Y. Hwang, "A Study on real-time fall detection with a smartphone, Chonbuk national Univ", master's theis Chonbuk national University, (2013).

[6] Y. Hirata, S. Komatsuda, K. Kosuge, "Fall prevention Control of Passive Intelligent Walker based on Human Model", IEEE/RSJ International Conference on Intelligent Robots and Systems Acropolis Convention Center, (2008), pp.1222-1228.

[7] Y.Kai, T.Tanioka, Y Inoue, "A walking Support/Evaluation Machine for Patients with parkinsonism", The Journal of Medical Inves-tigation, (2004), pp.117-124.

[8] J.M.Kim, H.K. Suh, "Risk Factors for Falls in the Elderly by Life-cycle", Korean Journal of Health Education and Promotion, vol. 27, no. 1, (2010).

[9] W.Y. Lee, D.K. Lee, M.S. Goh, E.H. Lee, "The Study of overcome the ramps for mobile walker", Proceeding of RESKO Technical Conference, (2011).

[10] M.S.Jang, I.S.Song, P.S.Kim, "A Study on Localization Algorithm using Hop Count and RSSI", International Journal of Control and Automation, (2013).

[11] J.H.Yoon, S.H.Kim, J.K.Oh, "The Effects of NoFalls Exercise Program on Fall Related Physical Fitness in Korean Elderly Women”, The Korea Journal of Sports Science, vol. 19, vol. 3, (2010), pp.1317-1325

[12] W.Y. Lee, "The Study on Control System for Walking Assistant Robot to Robust to the Road Surface Conditions, Korea Polytechnic University master's thesis. (2012)

[13] D.K. Lee, J.S.Kong, M.S.Goh, S.M.Lee, S.J.Kang, E.H.Lee, "Design of the Control Algorithm for Improvement of the Convenience the Active-type Walking Aid", Journal of Rehabilitation Welfare Engineering \& Assistive Technolygy, vol. 5, no. 1, (2011), pp. 17-25.

[14] S. J. Hall, "Basic Biomechanics", 5th Edition.

[15] D.A. Winter, "Human balance and posture control during standing and walking Gait\&Posture", (1995), pp.193-214.

[16] M.S. Jang, E.H. Lee, S.B. Choi, "A Study on Human Robot Interaction Technology Using a Circular Coordinate System for the Remote Control of the Mobile Robot", International Journal of Control and Automation, vol. 5, no. 4, (2012), pp.117-129.

[17] M.S.Jang, J.H.Shim, E.H.Lee, S.B.Choi, "Development of Motor, Encoder Evaluation System using High Performance DSP”, Journal of the Semiconductor \& Display Equiment Technology, vol. 8, no. 4, (2009), pp.77-82. 

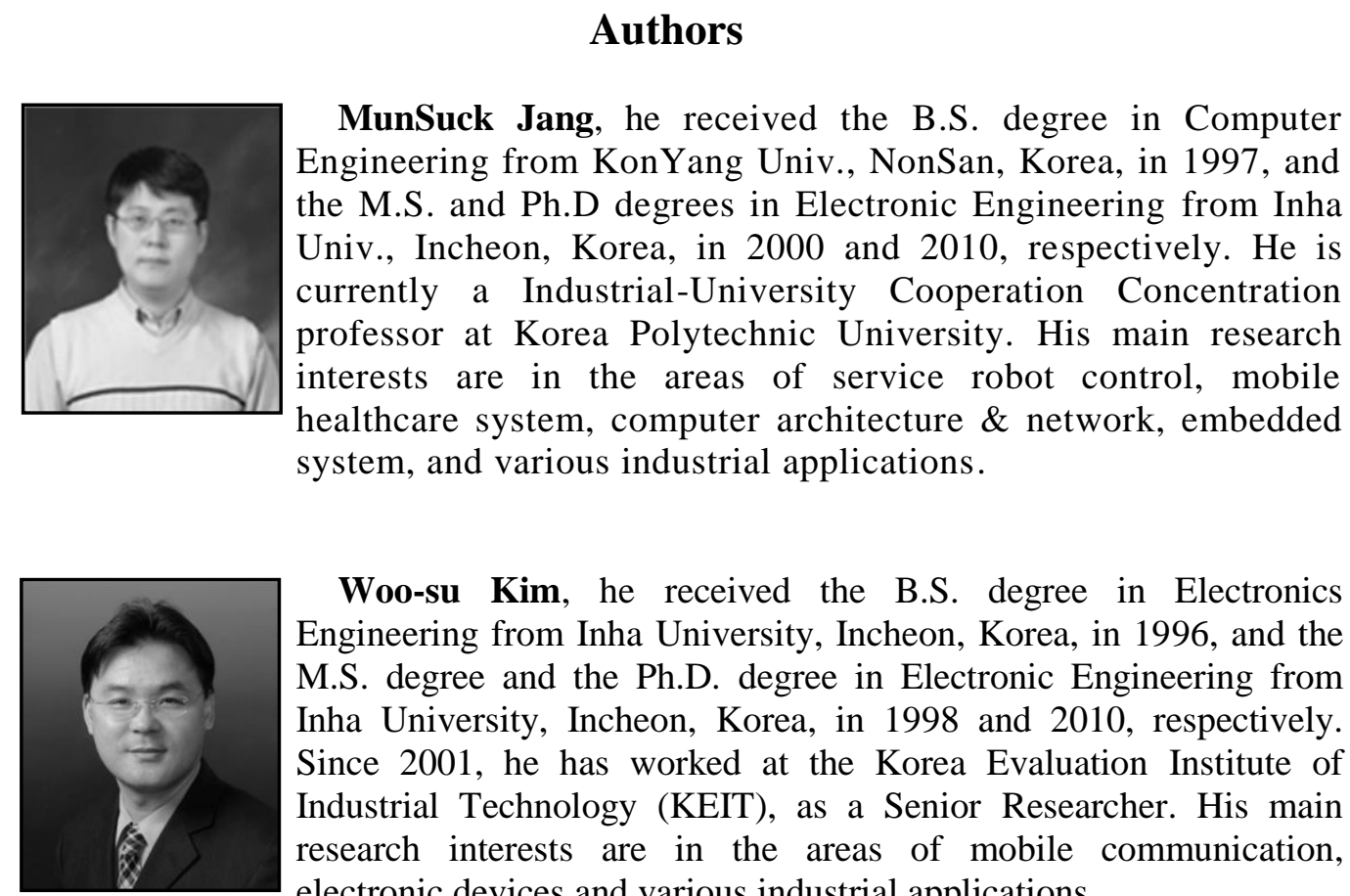

Woo-su Kim, he received the B.S. degree in Electronics Engineering from Inha University, Incheon, Korea, in 1996, and the M.S. degree and the Ph.D. degree in Electronic Engineering from Inha University, Incheon, Korea, in 1998 and 2010, respectively. Since 2001, he has worked at the Korea Evaluation Institute of Industrial Technology (KEIT), as a Senior Researcher. His main research interests are in the areas of mobile communication, electronic devices and various industrial applications.

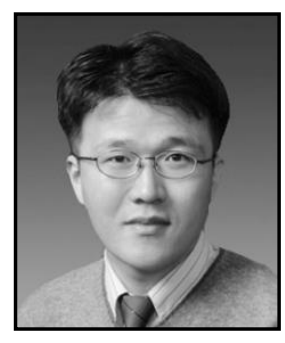

SeungJin Yoon, he received the B.S. degree and the M.S. degree in Computer Engineering from Chonbuk University, JeonJu, Korea, in 1999 and 2001, respectively, and the Ph.D. degree in Computer Engineering from Seokyeong University, Seoul, Korea, in 2012. From 2001 to 2002, he was a senior researcher at CDMA mobile lab. of LG Electronics Inc. Since 2002, he has been with the Korea Evaluation Institute of Industrial Technology. His main research interests are in the areas of robot control, communication networks and various industrial applications.

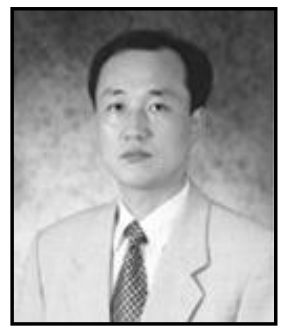

EungHyuk Lee, he received the B.S. degree in Electronics Engineering from Inha University, Incheon, Korea, in 1985, and the M.S. degree and the Ph.D. degree in Electronic Engineering from Inha University, Incheon, Korea, in 1985 and 1987, respectively. From 1987 to 1992, he was a researcher at Industrial Robot Lab. of Daewoo Heavy Industry Co. Ltd. From 1995 to 2000, he was a assistive professor at Dept. of Computer Engineering in KonYang University. Since 2000, he has been with the Department of Electronics Engineering at Korea Polytechnic University. His main research interests are in the areas of service robot control, mobile healthcare system, image processing and various industrial applications. 